\title{
Estrutura do estrato herbáceo na formação aberta de Clusia do Parque Nacional da Restinga de Jurubatiba, RJ, Brasil ${ }^{1}$
}

\author{
Miriam Cristina Alvarez Pereira ${ }^{2,5}$, Sandra Zorat Cordeiro ${ }^{2,3}$ e Dorothy Sue Dunn de Araujo ${ }^{2,4}$
}

Recebido em 30/09/2003. Aceito em 28/02/2004

\begin{abstract}
RESUMO - (Estrutura do estrato herbáceo na formação aberta de Clusia do Parque Nacional da Restinga de Jurubatiba, RJ, Brasil). No Parque Nacional da Restinga de Jurubatiba localiza-se o site 5 do Programa de Pesquisas Ecológicas de Longa Duração (PELD), região de grande diversidade de hábitats e riqueza florística nas restingas do Norte do Estado do Rio de Janeiro. Para descrever a composição florística e estrutura do estrato herbáceo da formação aberta de Clusia foram amostrados todos os indivíduos deste estrato utilizando-se o método de parcelas. Em três diferentes áreas foram distribuídos 200 quadrados de $1 \mathrm{~m}^{2}$, totalizando $600 \mathrm{~m}^{2}$. Em cada quadrado anotouse o número de indivíduos e percentagem de cobertura de cada espécie. Para a comparação entre as áreas utilizaram-se os índices de Similaridade de Sørensen, diversidade de Shannon (H') e equabilidade de Pielou (J'). Foram amostrados 39 espécies e 3.021 indivíduos. Allagoptera arenaria (Gomes) Kuntze, Vriesea neoglutinosa Mez, Aechmea nudicaulis (L.) Griseb., Stigmaphyllon paralias A. Juss., Neoregelia cruenta (Graham) L.B. Sm., Anthurium maricense Nadruz \& Mayo, Pilosocereus arrabidae (Lem.) Byles \& G.D. Rowl. e Ipomoea imperati (Vahl) Griseb. obtiveram os maiores valores de importância. A diversidade foi $\mathrm{H}^{\prime}=1,89$ nats $/ \mathrm{m}^{2}$ e a equabilidade $\mathrm{J}^{\prime}=0,52$, utilizando a cobertura como medida de abundância das espécies por esta evitar a subjetividade na definição de indivíduos e melhor representar a estrutura oligárquica aqui descrita. $\mathrm{O}$ ponto $\mathrm{A}$ diferencia-se significativamente de $\mathrm{B}$ e $\mathrm{C}$ quanto ao número de indivíduos e à cobertura vegetal herbácea. O número de espécies e os índices de diversidade não apresentaram diferenças significativas. Os resultados aqui apresentados diferem parcialmente dos obtidos anteriormente para o estrato arbustivo, sugerindo que estudos sobre distribuição espacial e associação entre espécies são necessários para esclarecer as relações entre estes dois estratos.
\end{abstract}

Palavras-chave: estrato herbáceo, restinga, estrutura oligárquica, PELD, Rio de Janeiro

\begin{abstract}
Herb layer structure of Clusia scrub in the Restinga de Jurubatiba National Park, Rio de Janeiro, Brazil). Long Term Ecological Research (LTER) Site No.5 in the Restinga de Jurubatiba National Park, northern Rio de Janeiro State protects a region rich in habitats and flora. We describe the variation in flora and structure of the herbaceous layer of the open Clusia scrub formation at three different sites near two coastal lagoons. All herb layer plants were sampled at each site in $2001 \times 1 \mathrm{~m}$ quadrats (total: $\left.600 \mathrm{~m}^{2}\right)$; number of plants and percent cover were recorded in each quadrat. These sites were compared as to similarity (Sørensen), diversity (Shannon: H') and evenness (J'). A total of 3,021 plants were sampled in 39 species, Allagoptera arenaria (Gomes) Kuntze, Vriesea neoglutinosa Mez, Aechmea nudicaulis (L.) Griseb., Stigmaphyllon paralias A. Juss., Neoregelia cruenta (Graham) L.B. Sm., Anthurium maricense Nadruz \& Mayo, Pilosocereus arrabidae (Lem.) Byles \& G.D. Rowl. and Ipomoea imperati (Vahl) Griseb. had the highest importance values. The diversity index was $\mathrm{H}^{\prime}=1.89$ nats $/ \mathrm{m}^{2}\left(\mathrm{~J}^{\prime}=0.52\right)$ based on plant cover in order to avoid having to determine what constituted an individual and because this parameter represented the oligarchic structure more precisely. Site A is significantly different from sites B and $\mathrm{C}$ in number of individuals and herbaceous cover, but not in number of species and diversity indices. Our results differ in part from those observed previously for the shrub layer, thus indicating a need for studies on spatial distribution and species associations in order to clarify the relationship between these two strata.
\end{abstract}

Key words: herb layer, sandy coastal plain, oligarchic structure, LTER, Brazil

\section{Introdução}

As restingas no Estado do Rio de Janeiro ocupam cerca de $1.200 \mathrm{~km}^{2} \mathrm{e}$ em seu litoral norte encontram-se as maiores áreas, destacando-se as regiões de São
João da Barra e Macaé (Araujo \& Maciel 1998), onde em 1998, foi criado o Parque Nacional da Restinga de Jurubatiba (PARNA da Restinga Jurubatiba), com a finalidade de proteger uma região de grande diversidade de hábitats e riqueza florística, incluindo espécies

\footnotetext{
1 Projeto "Perturbações naturais e antrópicas nos ecossistemas da restinga norte fluminense: sucessão estrutural e funcional como base para conservação e manejo" Site 5 do Programa de Pesquisas Ecológicas de Longa Duração (PELD)

2 Universidade Federal do Rio de Janeiro, Instituto de Biociências, Centro de Ciências da Saúde, Departamento de Ecologia, Laboratório de Ecologia Vegetal, C. Postal 68020, Ilha do Fundão, CEP 21941-590, Rio de Janeiro, RJ, Brasil

3 Bolsista FAPERJ Processo E26/151.257/01

4 Bolsista de Produtividade - CNPq

Autor para correspondência: miriamcap@ig.com.br
} 
ameaçadas de extinção.

A flora das restingas deste Estado é constituída, até o momento, por mais de 1.000 espécies, onde as ervas e subarbustos representam cerca de $25 \%$ (Araujo 2000). Em florestas tropicais Gentry \& Dodson (1987) observaram que o estrato herbáceo arbustivo é mais representativo que o arbóreo em relação ao número de espécies (35-52\% ×15-22\%). Porém, o entendimento do papel ecológico destas espécies ainda é incipiente. Discute-se, por exemplo, que espécies herbáceas possam ser utilizadas como indicadoras de condições ambientais, pois devido ao seu pequeno porte e sistema radicular superficial, estão submetidas a maior concorrência, tornando-se particularmente sensíveis às diferenças do ambiente (microclima e solo), às quais os vegetais de maior porte não manifestariam reação (Citadini-Zanette 1984; Citadini-Zanette \& Baptista 1989).

Embora as formações vegetais das restingas fluminenses tenham sido estudadas de forma mais intensa a partir da década de 1970, os trabalhos sobre estrutura de comunidades herbáceas são raros e normalmente realizados nas faixas de vegetação próximas à praia (e.g. Ormond 1960; Henriques et al. 1984; Assumpção \& Nascimento 1998; Menezes \& Araujo 1999) com algumas exceções como por exemplo no cordão arenoso interno na Reserva Ecológica de Jacarepiá, Saquarema (Sá 1996).

A formação aberta de Clusia estende-se por cerca de $40 \%$ da área da Restinga de Carapebus (Henriques et al. 1986) que está incluída no PARNA da Restinga de Jurubatiba. Esta se caracteriza por apresentar ilhas de vegetação intercaladas por áreas de areia branca, onde elevadas temperaturas, salinidade e solos pobres restringem a colonização via sementes. Tem sido proposto que plantas pioneiras e/ou facilitadoras (e.g., Allagoptera arenaria, Aechmea nudicaulis e Clusia hilariana) favorecem a entrada de outras espécies nesta comunidade (Scarano 2002; Zaluar \& Scarano 2000).

Em estudo realizado para descrever a estrutura do estrato arbustivo, em 12 pontos desta comunidade no PARNA Restinga de Jurubatiba, foram observadas poucas espécies dominantes caracterizando uma estrutura oligárquica, provavelmente resultante da atuação de fatores abióticos adversos e ação antrópica (Scarano 2002). Os valores de riqueza e diversidade de espécies entre os pontos não mostraram variação, mas quanto à cobertura vegetal houve diferença significativa. Portanto, são objetivos deste trabalho descrever florística e estruturalmente o estrato herbáceo da formação aberta de Clusia do Parque Nacional da Restinga de Jurubatiba; detectar variações em sua composição florística e estrutura; relacionar as variações observadas a diferenças de cobertura vegetal detectadas para o estrato arbustivo em Araujo et al. (2004); analisar a coexistência dos dois estratos na amostra aqui realizada.

\section{Material e métodos}

O Parque Nacional da Restinga de Jurubatiba abrange áreas dos municípios de Macaé, Carapebus e Quissamã (22 $2^{\circ}-22^{\circ} 23^{\prime} \mathrm{S}$; $41^{\circ} 15^{\prime}-41^{\circ} 45^{\prime} \mathrm{W}$, Fig. 1) com área total de $148,6 \mathrm{~km}^{2}$. A região é dominada por uma grande lagoa de água doce (Lagoa Feia) e por uma planície arenosa quaternária que se estende desde a cidade de Macaé até Barra do Furado (Araujo \& Maciel 1998; Araujo et al. 1998).

Os solos desta área são, na sua maioria, regossolos com baixo conteúdo de matéria orgânica e nutrientes, ocorrem também nesta região solos Hidromórficos, Orgânicos, Glei Úmico, Glei Pouco Úmico e Salinos.

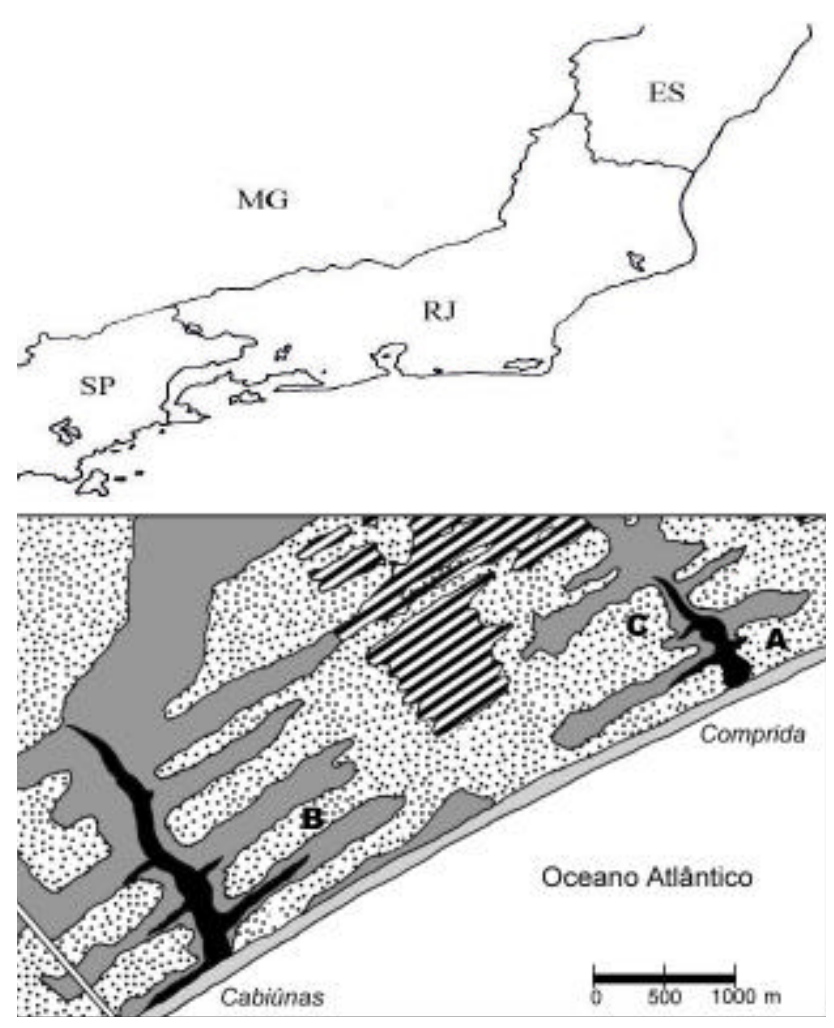

Figura 1. Localização do Parque Nacional da Restinga de Jurubatiba (PARNA Jurubatiba) no Estado do Rio de Janeiro. No detalhe, disposição dos pontos utilizados para a amostragem (A, B e C) na formação aberta de Clusia. Modificado de Mattos et al. (2004). . Restinga de Clusia; $\square$ Restinga de Ericaceae; $\square$ Matas; $\square$ Lagoas; $\square$ Praia. 
Segundo dados da estação meteorológica de Macaé, a precipitação anual é de $1.164 \mathrm{~mm}$, com distribuição fortemente sazonal, indicando pelo menos um período seco no mês de junho e entre os meses de junho e outubro o solo apresenta deficiência de água. A temperatura média anual é de $22,6^{\circ} \mathrm{C}$ (máxima $29,7^{\circ} \mathrm{C}$ em janeiro; mínima $20^{\circ} \mathrm{C}$ em julho) (Henriques et al. 1986).

Dentre as dez comunidades vegetais descritas para a área (Henriques et al. 1986; Araujo 1992; Araujo et al. 1998) a formação aberta de Clusia ocorre em extensa área do PARNA da Restinga de Jurubatiba e pode ser caracterizada por ocupar as regiões de topografia mais alta, ou seja, áreas onde o lençol freático não aflora; ser constituída por moitas densas, com forma esférica até totalmente irregulares, e tamanhos variados $\left(<1 \mathrm{~m}^{2}\right.$ até $\left.>1.000 \mathrm{~m}^{2}\right)$, intercaladas por espaços de areia onde a cobertura vegetal é esparsa ou constituída por pequenas moitas $\left(<5 \mathrm{~m}^{2}\right)$ onde predomina Allagoptera arenaria (Araujo et al. 1998; Zaluar \& Scarano 2000). O percentual de cobertura das moitas varia entre 39 e $45 \%$ (OliveiraGalvão et al. 1990). As espécies do estrato lenhoso que apresentam maiores valores de importância (VI) são: Clusia hilariana, Protium icicariba, Myrcia lundiana, Erythroxylum subsessile, Myrsine parvifolia e Ocotea notata (Araujo et al. 2004). A vegetação herbácea entre moitas apresenta cobertura vegetal entre 2-10\% (Oliveira-Galvão et al. 1990).

A partir de dados referentes ao estrato arbustivo, levantados em 12 diferentes pontos da comunidade aberta de Clusia do PARNA Jurubatiba e baseado nas variações observadas na sua cobertura vegetal (Araujo et al. 2002) foram escolhidos três pontos de

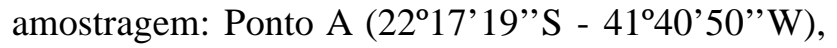

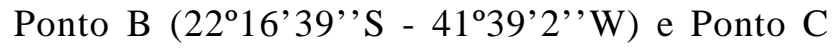

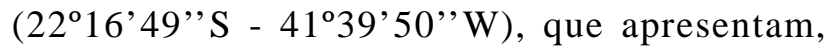
respectivamente, baixa, intermediária e alta cobertura vegetal do estrato arbustivo (Fig. 1).

Para a coleta de dados referentes às plantas do estrato herbáceo utilizou-se o método de parcelas (Müeller-Dombois \& Ellenberg 1974; Brower \& Zar 1984). Em cada ponto de coleta foram estabelecidas 200 parcelas permanentes de $1 \mathrm{~m}^{2}(1 \times 1 \mathrm{~m})$, alocadas de forma contígua em duas faixas de $1 \times 100 \mathrm{~m}\left(100 \mathrm{~m}^{2}\right)$, distantes entre si cerca de $200 \mathrm{~m}$ e perpendiculares à linha da costa, totalizando $600 \mathrm{~m}^{2}$ amostrados.

Em cada parcela de $1 \mathrm{~m}^{2}$ foram amostrados todos os indivíduos das espécies constituintes do estrato herbáceo (ervas + subarbustos, excluindo trepadeiras e epífitas) onde se levantaram os seguintes dados: número de espécies e indivíduos e percentagem de cobertura de cada espécie. Além disso, também foram anotadas as coberturas do estrato arbustivo, detritos e do espaço sem vegetação. Devido ao tamanho das unidades amostrais os dados aqui levantados para o estrato arbustivo foram utilizados apenas para avaliar a coexistência deste com o herbáceo.

A estimativa visual da cobertura foi realizada com o auxílio de um quadrado de $1 \times 1 \mathrm{~m}$ dividido com fios de nylon em 100 quadrados, correspondendo cada um a $1 \%$ da área. Com a finalidade de tornar mais precisa a estimativa visual do percentual de cobertura das espécies e devido às dificuldades de amostrar algumas destas por causa de sua morfologia e/ou forma de crescimento, estabeleceram-se os seguintes limites metodológicos: o percentual de cobertura mínimo para os indivíduos herbáceos foi de $1 \%$ e para os arbustos foi de $5 \%$; indivíduos não enraizados dentro da unidade amostral não foram considerados para o cálculo da densidade, no entanto, sua presença foi anotada e cobertura estimada para cálculo da freqüência e cobertura da espécie.

Foram incluídos na amostragem indivíduos das espécies arbustivas Gaylussacia brasiliensis, Vernonia rufogrisea e Waltheria aspera, porque estes ocorrem freqüientemente isolados nos espaços entre as moitas, normalmente, com altura inferior a $50 \mathrm{~cm}$. Tillandsia stricta, epífita herbácea, aparece na amostra por possuir alguns poucos indivíduos fixados ao solo ou sobre raízes superficiais.

As espécies de Bromeliaceae, Stigmaphyllon paralias e Ipomoea imperati apresentaram dificuldades na delimitação de seus indivíduos, portanto nestes casos foram contados seus rametes, com exceção de I. imperati onde se considerou a ocorrência de um indivíduo por parcela.

Os parâmetros quantitativos densidade, freqüência, cobertura absoluta e relativa, e valor de importância (VI) foram calculados segundo Brower \& Zar (1984).

Para comparar os três pontos e as seis faixas foram utilizados os números de indivíduos e espécies; a percentagem de coberturas herbácea, de detritos e de espaço sem vegetação; os índices de similaridade de Sørensen, Sørensen quantitativo, diversidade de Shannon e equabilidade entre espécies de Pielou, utilizando cobertura como medida de abundância (Magurran 1988). A similaridade entre as seis faixas também foi investigada através de análise de agrupamento pelo método de associação UPGMA (Valentin 2000) utilizando os índices de Sørensen. 
O teste do $x^{2}$ foi utilizado para verificar se as diferenças observadas quanto ao número de indivíduos e espécies, entre pontos ou faixas, eram significativas estatisticamente, e para a cobertura herbácea (o total de cada parcela de $1 \mathrm{~m}^{2}$ ) foi utilizado o teste KruskalWallis (ANOVA não paramétrica). Os índices de diversidade $\left(\mathrm{H}^{\prime}\right)$ foram comparados através do método de Hutcheson para o calculo do teste ' $t$ ' na comparação entre diversidades (Zar 1996).

As percentagens de coberturas do estrato herbáceo e arbustivo, nas 600 parcelas de $1 \mathrm{~m}^{2}$, foram usadas para estimar o coeficiente de correlação de Spearman - $\mathrm{r}_{\mathrm{s}}$ (Zar 1996).

A distribuição geográfica das espécies foi baseada na literatura especializada sobre os diversos táxons, sendo consultadas revisões, dissertações, teses, floras regionais e floras mais abrangentes (e.g., Flora Neotropica Monographs).

Todas as espécies foram coletadas e o material botânico conservado através das técnicas usuais de herborização. As exsicatas estão incluídas no acervo do herbário do Departamento de Botânica da UFRJ (RFA). O material foi identificado com o auxílio e comparação com material de herbário. Aidentificação definitiva do material foi realizada pelos respectivos especialistas de cada família botânica. Os autores das espécies estão de acordo com Brummitt \& Powell (1992).

\section{Resultados e discussão}

Estrutura e composição florística - A cobertura vegetal total do estrato herbáceo foi de $103 \mathrm{~m}^{2}(17 \%$ da área amostrada), detritos $245,5 \mathrm{~m}^{2}(41 \%)$, podendo haver sobreposição destas, e a percentagem de solo sem cobertura vegetal e/ou detritos foi $47 \%\left(284,2 \mathrm{~m}^{2}\right)$. A diferença entre os valores de cobertura vegetal herbácea aqui apresentados e os encontrados por Oliveira-Galvão et al. (1990) e Henriques et al. (1986) ocorreram porque nossa amostra é composta pelas plantas que ocupam tanto o espaço entre moitas como sob a cobertura arbustiva.

Foram observadas 39 espécies, distribuídas em 35 gêneros, pertencentes a 20 famílias nos $600 \mathrm{~m}^{2}$ amostrados (Tab. 1). As famílias com maior número de espécies são: Rubiaceae (5); Bromeliaceae, Cactaceae, Leguminosae (4); Orchidaceae e Poaceae (3); Araceae, Convolvulaceae e Solanaceae (2). Dentre estas, apenas Convolvulaceae não é observada entre as 20 famílias mais ricas em espécies nas restingas do Rio de Janeiro (Araujo 2000).
Nas parcelas foram amostrados 3.021 indivíduos; as famílias mais representativas foram Bromeliaceae, com 809 (27\% do total); Arecaceae - 487 (16\%), Malpighiaceae - 339 (11\%), Araceae - 293 (10\%), Rubiaceae - 204 (7\%), Cactaceae - 202 (7\%), Leguminosae - 164 (5\%) e Convolvulaceae 147 (5\%). Estas totalizam $87 \%$ do número total de indivíduos.

A Tabela 1 apresenta os parâmetros quantitativos para as 39 espécies amostradas, listadas em ordem decrescente de VI. As famílias com maiores VIs foram Arecaceae $(88,9)$ e Bromeliaceae $(69,1)$, somando 53\% do total. A importância de Arecaceae deve-se a uma única espécie - Allagoptera arenaria - que obteve os maiores valores relativos de densidade, frequiência e, principalmente, cobertura na amostra. Malpighiaceae também destaca-se dentre as famílias dominantes devido à importância de uma única espécie Stigmaphyllon paralias. Outras famílias como Bromeliaceae, Cactaceae, Convolvulaceae e, com menores valores, Leguminosae e Rubiaceae, também, são importantes na estrutura da comunidade.

Embora as espécies de Bromeliaceae tenham tido sua densidade superestimada devido à contagem de rametes, sua importância para estrutura deste estrato é confirmada por elevados valores de cobertura, principalmente no caso de Vriesea neoglutinosa. Por outro lado o alto VI de Stigmaphyllon paralias deve-se à sua freqüência, que pode ter sido superestimada devido ao crescimento clonal.

As Cactaceae, em especial Pilosocereus arrabidae, destacam-se quanto às frequiências relativas, assim como as Convolvulaceae. A estratégia utilizada para a contagem de indivíduos de Ipomoea imperati não superestimou sua densidade, uma vez que esta obteve elevado VI devido à frequiência relativa.

Oito espécies foram consideradas dominantes por possuírem VI maior que 10,0 e por representarem 74\% do total deste parâmetro, 21 apresentaram VI entre 10,0 e 1,0 , somando $24 \%$ do total, sendo aqui denominadas espécies intermediárias e as 10 raras, $\mathrm{VI}<1,0$, totalizaram $2 \%$. Esta dominância de poucas espécies caracteriza estrutura oligárquica para a comunidade em estudo.

A espécie de maior VI, Allagoptera arenaria, destaca-se por sua elevada cobertura relativa (51\%), sendo que em outras restingas do Estado do Rio de Janeiro o valor deste parâmetro também pode ser alto, variando entre 30 e $79 \%$ (Almeida \& Araujo 1997; Araujo \& Peixoto 1977; Menezes \& Araujo 1999). A maioria de seus indivíduos é encontrada sobre a areia nua nos espaços entre as moitas ou formando pequenas 
Tabela 1. Espécies do estrato herbáceo e seus parâmetros fitossociológicos organizadas em ordem decrescente de VI, nas áreas estudadas da Formação aberta de Clusia do Parque Nacional da Restinga de Jurubatiba. N - número de indivíduos, A- número de unidades amostrais $\left(1 \mathrm{~m}^{2}\right)$ com a presença da espécie, C - Cobertura, D - Densidade, DR - Densidade relativa, F - Freqüência, FR - Freqüência relativa, CR - Cobertura relativa e VI - Valor de Importância.

\begin{tabular}{|c|c|c|c|c|c|c|c|c|c|c|}
\hline Espécie & Família & $\mathrm{N}$ & A & $\begin{array}{l}\mathrm{C} \\
\left(\mathrm{m}^{2}\right)\end{array}$ & $\begin{array}{l}\mathrm{D} \\
\left(\mathrm{m}^{2}\right)\end{array}$ & $\begin{array}{l}\mathrm{DR} \\
(\%)\end{array}$ & $\mathrm{F}$ & $\begin{array}{l}\mathrm{FR} \\
(\%)\end{array}$ & $\begin{array}{l}\mathrm{CR} \\
(\%)\end{array}$ & $\begin{array}{l}\mathrm{VI} \\
(\%)\end{array}$ \\
\hline Allagoptera arenaria (Gomes) Kuntze & Arecaceae & 487 & 363 & 52,53 & 0,812 & 16,12 & 0,605 & 21,84 & 50,95 & 88,91 \\
\hline Vriesea neoglutinosa $\mathrm{Mez}$ & Bromeliaceae & 210 & 27 & 17,00 & 0,350 & 6,95 & 0,045 & 1,62 & 16,49 & 25,06 \\
\hline Aechmea nudicaulis (L.) Griseb. & Bromeliaceae & 358 & 89 & 5,95 & 0,597 & 11,85 & 0,148 & 5,35 & 5,77 & 22,98 \\
\hline Stigmaphyllon paralias A. Juss. & Malpighiaceae & 339 & 130 & 1,7 & 0,565 & 11,22 & 0,217 & 7,82 & 1,65 & 20,69 \\
\hline Neoregelia cruenta (Graham) L.B. Sm. & Bromeliaceae & 225 & 93 & 7,03 & 0,375 & 7,45 & 0,155 & 5,60 & 6,82 & 19,86 \\
\hline Anthurium maricense Nadruz \& Mayo & Araceae & 288 & 80 & 2,17 & 0,480 & 9,53 & 0,133 & 4,81 & 2,10 & 16,45 \\
\hline $\begin{array}{l}\text { Pilosocereus arrabidae (Lem.) Byles \& } \\
\text { G.D. Rowl. }\end{array}$ & Cactaceae & 153 & 118 & 4,42 & 0,255 & 5,06 & 0,197 & 7,10 & 4,29 & 16,45 \\
\hline Ipomoea imperati (Vahl) Griseb. & Convolvulaceae & 113 & 113 & 1,21 & 0,188 & 3,74 & 0,188 & 6,80 & 1,17 & 11,71 \\
\hline $\begin{array}{l}\text { Diodia apiculata (Roem. \& Schult.) } \\
\text { K. Schum. }\end{array}$ & Rubiaceae & 75 & 63 & 0,9 & 0,125 & 2,48 & 0,105 & 3,79 & 0,87 & 7,15 \\
\hline Mollugo verticillata $\mathrm{L}$. & Molluginaceae & 86 & 52 & 0,65 & 0,143 & 2,85 & 0,087 & 3,13 & 0,63 & 6,61 \\
\hline Mitracarpus frigidus (Willd.) K. Schum. & Rubiaceae & 95 & 46 & & 0,1 & 3,14 & & 2,77 & 0,45 & 6,36 \\
\hline $\begin{array}{l}\text { Chamaecrista ramosa (Vogel) H.S. Irwin } \\
\text { \& Barneby }\end{array}$ & Leguminosae & 46 & 52 & 0,96 & 0,077 & 1,52 & 0,087 & 3,13 & 0,93 & 5,58 \\
\hline Stylosanthes viscosa $\mathrm{Sw}$ & Leguminosae & 53 & 37 & 0,47 & 0,088 & 1,75 & 0,062 & 2,23 & 0,46 & 4,44 \\
\hline Evolvulus genistoides Ooststr. & Convolvulaceae & 34 & 40 & 0,88 & 0,057 & 1,13 & 0,067 & 2,41 & 0,85 & 4,39 \\
\hline Cuphea flava Spreng. & Lythraceae & 42 & 33 & 0,76 & 0,070 & 1,39 & 0,055 & 1,99 & 0,74 & 4,11 \\
\hline Chamaecrista flexuosa $\mathrm{L}$. & Leguminosae & 35 & 37 & 0,37 & 0,058 & 1,16 & 0,062 & 2,23 & 0,36 & 3,74 \\
\hline Schwenckia americana $\mathrm{L}$. & Solanaceae & 53 & 22 & 0,32 & 0,088 & 1,75 & 0,037 & 1,32 & 0,31 & 3,39 \\
\hline Stylosanthes guianensis Sw. & Leguminosae & 30 & 32 & 0,44 & 0,050 & 0,99 & 0,053 & 1,93 & 0,43 & 3,35 \\
\hline $\begin{array}{l}\text { Gaylussacia brasiliensis (Spreng.) } \\
\text { Meisn. }\end{array}$ & Ericaceae & 60 & 16 & 0,37 & 0,100 & 1,99 & 0,027 & 0,96 & 0,36 & 3,31 \\
\hline Chioccoca alba (L.) Hitchc. & Rubiaceae & 24 & 32 & 0,52 & 0,040 & 0,79 & 0,053 & 1,93 & 0,50 & 3,22 \\
\hline Cereus fernambucensis Lem. & Cactaceae & 27 & 28 & 0,65 & 0,045 & 0,89 & 0,047 & 1,68 & 0,63 & 3,21 \\
\hline Pteridium aquilinum (L.) Kuhn. & Dennstaedtiaceae & 20 & 23 & 1,06 & 0,033 & 0,66 & 0,038 & 1,38 & 1,03 & 3,07 \\
\hline $\begin{array}{l}\text { Cyrtopodium polyphyllum (Vell.) Pabst } \\
\text { ex. Barros }\end{array}$ & Orchidaceae & 32 & 22 & 0,5 & 0,053 & 1,06 & 0,037 & 1,32 & 0,48 & 2,87 \\
\hline $\begin{array}{l}\text { Selenicereus setaceus (Salm-Dyck ex } \\
\text { DC.) Werderm. }\end{array}$ & Cactaceae & 19 & 17 & 0,24 & 0,032 & 0,63 & 0,028 & 1,02 & 0,23 & 1,88 \\
\hline Chamaecyse thymifolia (L.) Millsp. & Euphorbiaceae & 16 & 13 & 0,13 & 0,027 & 0,53 & 0,022 & 0,78 & 0,13 & 1,44 \\
\hline Gymnopogon foliosus (Willd.) Nees & Poaceae & 16 & 12 & 0,12 & 0,027 & 0,53 & 0,020 & 0,72 & 0,12 & 1,37 \\
\hline Tillandsia stricta Sol. & Bromeliaceae & 16 & 9 & 0,17 & 0,027 & 0,53 & 0,015 & 0,54 & 0,16 & 1,24 \\
\hline Epidendrum denticulatum Barb. Rodr. & Orchidaceae & 12 & 10 & 0,22 & 0,020 & 0,40 & 0,017 & 0,60 & 0,21 & 1,21 \\
\hline Aristida setifolia Kunth & Poaceae & 9 & 10 & 0,12 & 0,015 & 0,30 & 0,017 & 0,60 & 0,12 & 1,02 \\
\hline Vanilla cf. chamissonis Klotzsch & Orchidaceae & 8 & 7 & 0,21 & 0,013 & 0,26 & 0,012 & 0,42 & 0,20 & 0,89 \\
\hline Vernonia rufogrisea A. St.-Hil. & Asteraceae & 6 & 7 & 0,21 & 0,010 & 0,20 & 0,012 & 0,42 & 0,20 & 0,82 \\
\hline Solanum insidiosum Mart. & Solanaceae & 8 & 7 & 0,07 & 0,013 & 0,26 & 0,012 & 0,42 & 0,07 & 0,75 \\
\hline Borreria verticillata (L.) Meyer & Rubiaceae & 6 & 7 & 0,08 & 0,010 & 0,20 & 0,012 & 0,42 & 0,08 & 0,70 \\
\hline Waltheria aspera K. Schum. & Sterculiaceae & 4 & 4 & 0,04 & 0,007 & 0,13 & 0,007 & 0,24 & 0,04 & 0,41 \\
\hline Anthurium olfersianum Kunth & Araceae & 5 & 2 & 0,09 & 0,008 & 0,1 & 0,0 & 0,12 & 0,09 & 0,37 \\
\hline Melocactus violaceus Pfeiff. & Cactaceae & 3 & 3 & 0,03 & 0,005 & 0,10 & & 0,18 & 0,03 & 0,31 \\
\hline Polypodium triseriale $\mathrm{Sw}$. & Polypodiaceae & 3 & 3 & 0,03 & 0,005 & 0,10 & 0,005 & 0,18 & 0,03 & 0,31 \\
\hline Mitracarpus lhotzkyanus Cham. & Rubiaceae & 4 & 2 & 0,02 & 0,007 & 0,13 & 0,003 & 0,12 & 0,02 & 0,27 \\
\hline Poaceae 1 & Poaceae & 1 & 1 & 0,01 & 0,002 & 0,03 & 0,002 & 0,06 & 0,01 & 0,10 \\
\hline Totais & & 3.021 & & 103,11 & 5,035 & 100 & 2,770 & 100 & 100 & 300 \\
\hline
\end{tabular}

moitas, conforme já descrito para esta mesma comunidade do PARNA Restinga de Jurubatiba (Henriques et al. 1986; Araujo et al. 1998).

Esta palmeira geófita é uma das poucas espécies que consegue germinar na areia nua da restinga (Leite 1990, apud Zaluar \& Scarano 2000), e depois de queimada apresenta crescimento rápido, obtendo altos valores de cobertura relativa (Araujo \& Peixoto 1977; Cirne et al. 2003). Na formação aberta de Clusia do PARNA da Restinga de Jurubatiba, atua como espécie chave para a estrutura e funcionamento desta, juntamente com outras espécies de bromélia tanque, 
cactáceas e clusias (Scarano 2002).

Quanto às demais espécies dominantes, Stigmaphyllon paralias é citada por Henriques et al. (1986) como abundante na vegetação entre moitas da formação de Clusia em Carapebus. Estes autores, ainda, destacam que Aechmea nudicaulis e Neoregelia cruenta são encontradas em maior densidade fora das moitas. Os dados aqui levantados confirmam estas informações.

Ipomoea imperati é a única espécie dominante que não ocorreu nos três pontos estudados, estando presente na amostra apenas no cordão mais próximo ao mar (ponto A - baixa cobertura do estrato arbustivo). Esta espécie é característica da formação psamófila reptante em varias restingas do Estado do Rio de Janeiro (Almeida \& Araujo 1997; Assumpção \& Nascimento 1998; Menezes \& Araujo 1999). Outras espécies bem representadas quanto ao IVC no estudo de Assumpção \& Nascimento (2000) - Chamaecyse thymifolia, principalmente, e Mollugo verticillata - aqui são espécies intermediárias que não ocorrem no cordão mais interno (alta cobertura do estrato arbustivo). Essas três espécies seriam características de formações mais abertas próximas à praia.

Distribuição geográfica das espécies - Quanto à distribuição geográfica das espécies dominantes, Aechmea nudicaulis, Ipomoea imperati e Stigmaphyllon paralias possuem distribuição ampla. I. imperati ocorre somente em ambientes praianos, ou seja, é uma especialista, e A. nudicaulis tem ampla distribuição nos trópicos, podendo ser considerada uma generalista. Stigmaphyllon paralias está ausente da Floresta Atlântica sensu strictu, ocorrendo em ambientes mais secos como cerrado, campos rupestres e caatinga. As demais apresentam distribuição bastante restrita, na faixa costeira que se estende do sul da Bahia até Rio Grande do Sul, sendo que Neoregelia cruenta e Anthurium maricense são endêmicas nas restingas. A. maricense tem distribuição restrita ao Estado do Rio de Janeiro e $N$. cruenta ocorre neste Estado e no Espírito Santo (Araujo 2000). As outras espécies, Allagoptera arenaria, Pilosocereus arrabidae e Vriesea neoglutinosa são encontradas fora das restingas em ambientes abertos e próximos ao mar, ou seja, muito diferente do habitat típico da Floresta Atlântica s.s. Então, seis das oito espécies dominantes (75\%) podem ser consideradas especialistas.

Dentre as intermediárias, $16(76 \%)$ são generalistas e possuem ampla distribuição geográfica nos trópicos e/ou foram consideradas ruderais (Chamaecrista flexuosa, Chamaecyse thymifolia,
Mitracarpus frigidus, Mollugo verticillata, Pteridium aquilinum, Stylosanthes guianensis e S. viscosa) (Araujo 2000). Não foram encontradas informações sobre a distribuição geográfica das outras espécies intermediárias e raras.

A relação entre abundância local de espécies e área de distribuição geográfica foi observada por vários autores e pode ser positiva, negativa ou nula (Gaston \& Lawton 1990). Os dados apresentados acima mostram que $75 \%$ das espécies dominantes são especialistas, enquanto pelo menos $76 \%$ das espécies intermediárias são generalistas com distribuição ampla, o que representa relação negativa. De acordo com Gaston \& Lawton (1990), isto ocorre com maior frequiência em situações onde o hábitat de referência (i.e., onde as abundâncias foram estimadas) é muito diferente, no que se refere às condições climáticas ou recursos disponíveis, do hábitat mais comum na região geográfica como um todo. Aqui, o hábitat de referência é a formação aberta de Clusia da planície arenosa costeira norte-fluminense e o hábitat que prevalece na região é o da Floresta Atlântica sensu strictu A relação negativa entre abundância local de espécies e área de distribuição geográfica observada pode ser explicada da seguinte maneira segundo Gaston \& Lawton (1990): as especialistas tem alta abundância nas restingas, mas não conseguem sobreviver na Floresta Atlântica sensu strictu e por isso não possuem distribuição geográfica ampla; já as generalistas, que exploram ampla gama de hábitats, conseguem se manter no hábitat atípico (restinga), mas apenas com abundâncias baixas.

Entretanto, em hábitats marginais à Floresta Atlântica as diferenças entre generalistas e especialistas, quanto aos seus mecanismos de "fitness" e sobrevivência, não são marcantes (Scarano et al. 2001), e nestes a riqueza de espécies é mais baixa que na Floresta Atlântica, mas a diversidade ecofisiológica é alta. Segundo aqueles autores, o fato da planta ser generalista não garante que irá colonizar áreas geográficas mais amplas e, paradoxalmente, pode ter sido um mecanismo especializado (CAM) que permitiu a algumas espécies expandir suas distribuições. Desta maneira, a relação entre abundância local de espécies e área de distribuição geográfica nestes hábitats sujeitos a estresse ainda necessita de estudos mais detalhados, incluindo na análise um maior número de espécies.

Comparação entre as três áreas amostradas Comparando os três pontos amostrados (Tab. 2) verifica-se que existe diferença significativa quanto à cobertura vegetal herbácea $(\mathrm{p}<0,0001)$ e número de 
Tabela 2. Comparação entre os dados levantados em cada ponto amostrado $\left(200 \mathrm{~m}^{2}\right)$ da formação aberta de Clusia no Parque Nacional da Restinga de Jurubatiba. Em cada linha letras diferentes indicam diferenças significativas.

\begin{tabular}{lccc}
\hline & Ponto A & Ponto B & Ponto C \\
\hline Cobertura herbácea $\left(\mathrm{m}^{2}\right)$ & $22,5^{\mathrm{a}}$ & $40,8^{\mathrm{b}}$ & $39,7^{\mathrm{b}}$ \\
Cobertura Allagoptera arenaria $\left(\mathrm{m}^{2}\right)$ & 10,6 & 20,5 & 21,4 \\
Detritos $\left(\mathrm{m}^{2}\right)$ & 38,8 & 100,1 & 106,6 \\
Espaço sem vegetação $\left(\mathrm{m}^{2}\right)$ & 141,7 & 73,7 & 68,8 \\
Número de indivíduos & $917^{\mathrm{a}}$ & $1071^{\mathrm{b}}$ & $1033^{\mathrm{b}}$ \\
Número de espécies & 27 & 31 & 29 \\
Diversidade $\left(\mathrm{H}^{\prime}\right)$ & 1,99 & 1,75 & 1,77 \\
Equabilidade (J') & 0,64 & 0,51 & 0,52 \\
\hline
\end{tabular}

indivíduos $(\mathrm{P}<0,05)$ entre o ponto $\mathrm{A}$ (baixa cobertura vegetal arbustiva) e os demais; já entre $\mathrm{B}$ e $\mathrm{C}$ não há diferença significativa. No entanto, a menor cobertura pode ter influenciado os índices de diversidade e equiabilidade um pouco mais elevados em A. Na Tabela 2 observa-se que Allagoptera arenaria contribui com aproximadamente $50 \%$ da cobertura total em cada um dos três pontos, mas em A sua cobertura acompanha a redução observada para o valor total. Isto determinou maior eqüabilidade entre espécies, influenciando H', visto que o número de espécies comuns entre os três pontos variou pouco (20 a 23). O número de espécies e os índices de diversidade não apresentaram diferenças significativas entre os três pontos.

Normalmente os índices de diversidade de Shannon (H') e eqüabilidade de Pielou (J') não são apresentados nos trabalhos sobre estrutura do estrato herbáceo devido à dificuldade de distinguir indivíduos em espécies estoloníferas reptantes ou com crescimento clonal, que são comuns no estrato herbáceo de formações vegetais de restinga. No entanto, segundo Magurran (1988), nestes casos a biomassa, cobertura ou freqüência podem substituir o número de indivíduos como medida de abundância.

Ao comparar os índices calculados a partir do número de indivíduos $\left(\mathrm{H}_{\mathrm{I}}=2,88\right.$ nats/ind. e eqüabilidade $\left.\mathrm{J}_{\mathrm{I}}{ }_{\mathrm{I}}=0,78\right)$, cobertura $\left(\mathrm{H}^{\prime}{ }_{\mathrm{C}}=1,89\right.$ nats $\left./ \mathrm{m}^{2} \mathrm{e} \mathrm{J}{ }_{\mathrm{C}}=0,52\right)$ e frequiência $\left(\mathrm{H}_{\mathrm{F}}^{\prime}=3,01 \mathrm{e} \mathrm{J}_{\mathrm{F}}=0,82\right)$ observa-se que os maiores valores foram obtidos quando se utilizou a freqüência como medida de abundância, o quê, segundo Magurran (1988), pode subestimar a abundância das espécies mais comuns, aumentando a equabilidade entre as espécies, como foi aqui observado.

Os resultados de Müller (1999) para a sinúsia herbácea de uma floresta de restinga da planície costeira do Rio Grande do Sul também apresentam menores $\mathrm{H}_{\mathrm{C}}{ }_{\mathrm{C}}=1,97 \mathrm{e} \mathrm{J}_{\mathrm{C}}=0,61 \times$ maiores $\mathrm{H}_{\mathrm{F}}=2,51$ e $\mathrm{J}_{\mathrm{F}}{ }_{\mathrm{F}}=0,78$. Apesar de apresentar menor número de espécies (26), seus índices $\mathrm{H}_{\mathrm{C}} \mathrm{e} \mathrm{J}_{\mathrm{C}}{ }_{\mathrm{C}}$ foram superiores aos aqui obtidos, devido à menor desproporção entre a cobertura das espécies.

Detendo-se as espécies dominantes (Tab. 1) nota-se maior desproporção entre os valores obtidos para cobertura que para número de indivíduos, por isso obtiveram-se valores mais baixos quando se utilizou a cobertura como medida de abundância no cálculo de H'. Mesmo assim, esta parece ser a melhor opção para substituir o número de indivíduos por melhor representar a estrutura oligárquica aqui descrita e uma vez que a freqüência também pode ser influenciada pela subjetividade em distinguir indivíduos.

Quanto à similaridade florística os valores do coeficiente de Sørensen (Tab. 3) são altos (maiores que $70 \%$ ) e muito próximos entre A, B e C. Levando-se em consideração a abundância das espécies, isto é Sørensen quantitativo, notam-se menores graus de similaridade nas comparações entre A e os demais, e alta similaridade entre B e C. Como floristicamente os pontos não se diferenciam, apenas através da estrutura foi possível perceber que o ponto A se destaca dos demais, e que B e C são semelhantes.

$\mathrm{Na}$ Tabela 4 podem-se observar os resultados obtidos para cada faixa de $100 \mathrm{~m}^{2}$. Existe diferença significativa $(\mathrm{P}<0,05)$ quanto ao número de indivíduos, inclusive entre faixas de um mesmo ponto. No caso da cobertura herbácea a diferença é significativa entre as faixas do ponto $\mathrm{A}$ e as demais $(\mathrm{p}<0,0001)$. O mesmo não acontece em relação ao número de espécies e entre os índices de diversidade.

As seis faixas exibem similaridade florística entre si, sendo que o menor valor obtido $(0,65)$ foi entre o grupo formado pelas faixas do ponto A e outro formado pelas faixas de B e C (Fig. 2A). Os mesmos resultados

Tabela 3. Similaridade (Coeficientes de Sørensen e Sørensen Quantitativo) entre as áreas amostradas e para o estrato herbáceo da formação aberta de Clusia no Parque Nacional da Restinga de Jurubatiba.

\begin{tabular}{lccc}
\hline Sørensen & & Ponto B & Ponto C \\
\hline & Ponto A & 0,76 & $\begin{array}{l}0,71 \\
0,77\end{array}$ \\
\hline Ponto B & & Ponto C \\
\hline Sørensen Quantitativo & & Ponto B & 0,64 \\
& & 0,61 & 0,85 \\
\hline
\end{tabular}


Tabela 4. Comparação entre os dados levantados em cada faixa amostrada $\left(100 \mathrm{~m}^{2}\right)$ da formação aberta de Clusia no Parque Nacional da Restinga de Jurubatiba. Em cada linha letras diferentes indicam diferenças significativas.

\begin{tabular}{|c|c|c|c|c|c|c|}
\hline \multirow[b]{2}{*}{ Faixas } & \multicolumn{2}{|c|}{ Ponto A } & \multicolumn{2}{|c|}{ Ponto B } & \multicolumn{2}{|c|}{ Ponto C } \\
\hline & 3 & 4 & 1 & 2 & 5 & 6 \\
\hline Cobertura herbácea $\left(\mathrm{m}^{2}\right)$ & $11,6^{\mathrm{a}}$ & $10,9^{\mathrm{a}}$ & $23,5^{\mathrm{b}}$ & $17,3^{\mathrm{b}}$ & $17,5^{\mathrm{b}}$ & $22,2^{\mathrm{b}}$ \\
\hline Detritos $\left(\mathrm{m}^{2}\right)$ & 20,1 & 18,7 & 60,2 & 39,9 & 53,6 & 53,0 \\
\hline Espaço sem vegetação $\left(\mathrm{m}^{2}\right)$ & 69,0 & 72,7 & 27,4 & 46,3 & 34,5 & 34,3 \\
\hline Númerode espécies & 20 & 24 & 21 & 26 & 23 & 25 \\
\hline Número de indivíduos & $493^{\mathrm{a}}$ & $424^{c}$ & $485^{\mathrm{a}}$ & $586^{\mathrm{b}}$ & $410^{c}$ & $623^{b}$ \\
\hline Diversidade $\mathrm{H}^{\prime}$ & 1,93 & 1,87 & 1,62 & 1,50 & 1,46 & 1,82 \\
\hline Equabilidade J' & 0,65 & 0,59 & 0,53 & 0,46 & 0,46 & 0,57 \\
\hline
\end{tabular}

são observados quando se utiliza Sørensen quantitativo, tanto usando o número de indivíduos como a cobertura como medidas de abundância (Fig. 2B e C). Neste último caso nota-se que os maiores valores de similaridade foram obtidos entre pares de faixas de pontos diferentes (B e C). Isto demonstra, mais uma vez, que em relação ao número de indivíduos e cobertura, o ponto A se diferencia dos demais, e que B e C são semelhantes.

$\mathrm{O}$ ponto $\mathrm{A}$, ao contrário dos pontos $\mathrm{B}$ e $\mathrm{C}$, está localizado em área da restinga classificada por sensoriamento remoto como "Clusia clara" (OliveiraGalvão et al. 1990), ou seja, possui padrão tonal diferente de outras áreas classificadas como "Clusia escura" (onde estão localizados os pontos B e C). Segundo estes autores, "Clusia clara" apresenta menores densidades e dimensões de moitas e menor cobertura herbácea entre as moitas. Em imagens de satélite (Embrapa Monitoramento por Satelite), esta área estende-se da Lagoa Comprida até além da Lagoa de Carapebus, nas cristas de praia próximas ao mar. Aparentemente não teria existido influência mais intensa de atividades antrópicas, inclusive esta área localiza-se em trecho da planície de mais difícil acesso. O tamanho e posição da área ocupada pela classe "Clusia clara" sugere evolução paleogeográfica, discutida em Martin et al. (1993), possivelmente distinta do restante da planície arenosa, podendo estar relacionada, também, a algum fator abiótico atual como, por exemplo, maior exposição à influência marinha.

Estrato arbustivo $\times$ herbáceo - Os resultados aqui apresentados corroboram parcialmente aos obtidos para o estrato arbustivo (Araujo et al. 2004). Estes são similares no que se refere a número de espécies e índice de diversidade, não apresentando diferenças significativas entre os pontos. Porém, apenas o ponto

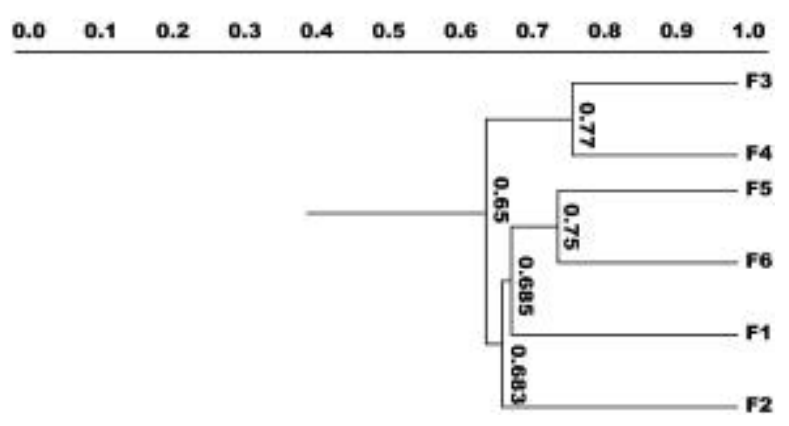

B

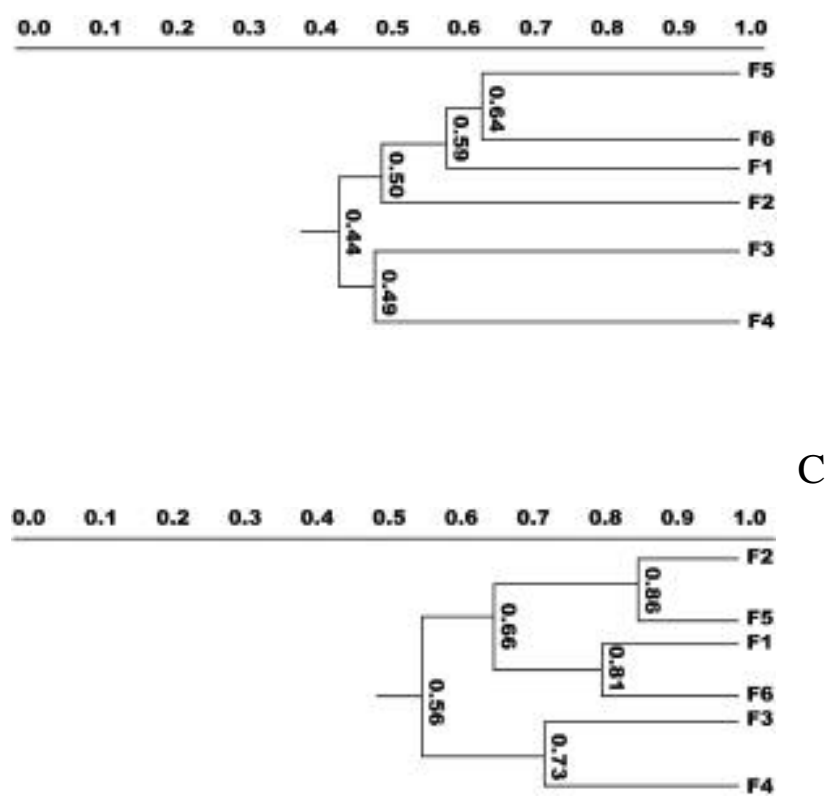

Figura 2. Similaridade entre as seis faixas utilizadas no levantamento de dados referentes ao estrato herbáceo da formação aberta de Clusia do Parque Nacional da Restinga de Jurubatiba, método de agrupamento UPGMA. A. Similaridade florística (Sørensen qualitativo). B. Similaridade estrutural (Sørensen quantitativo) baseada no número de indivíduos como medida de abundância. C. Similaridade estrutural (Sørensen quantitativo) baseada na cobertura como medida de abundância. 
A do estrato herbáceo possui baixa cobertura vegetal (Tab. 2) enquanto que no estrato arbustivo os pontos $\mathrm{A}, \mathrm{B}$ e $\mathrm{C}$ foram diferenciados, respectivamente, como de baixa, intermediária e alta coberturas. O número de indivíduos do estrato herbáceo apresentou a mesma variação observada para sua cobertura entre os pontos (Tab. 2), mas mostrou diferença significativa entre faixas de um mesmo ponto (Tab. 4).

Fisionomicamente a formação aberta de Clusia apresenta pelo menos dois segmentos ou estandes distintos: as áreas de areia com vegetação herbácea esparsa e as ilhas de vegetação, essas em diferentes estádios de desenvolvimento. Esta heterogeneidade de hábitats deve influenciar a composição e a estrutura do estrato herbáceo, a interação entre suas espécies, e ainda, sua coexistência com a vegetação arbustiva.

Sendo assim, muitas unidades amostrais (341 ou $57 \%$ do total) não apresentavam cobertura arbustiva e dentre estas, 237 tinham no máximo $10 \%$ de cobertura herbácea (Fig. 3). Estas parcelas representam as áreas de areia com vegetação herbácea esparsa entre ilhas de vegetação, e a baixa cobertura observada indica que nestas existe dificuldade de estabelecimento tanto para as plantas arbustivas quanto para as herbáceas.

Isto certamente influenciou a análise de coexistência dos dois estratos, onde foi constatada baixa correlação significativa entre as percentagens de cobertura dos estratos herbáceo e arbustivo nas 600 parcelas de $1 \mathrm{~m}^{2}\left(r_{\mathrm{s}}=0,36, \mathrm{P}<0,001\right.$; Fig. 3).

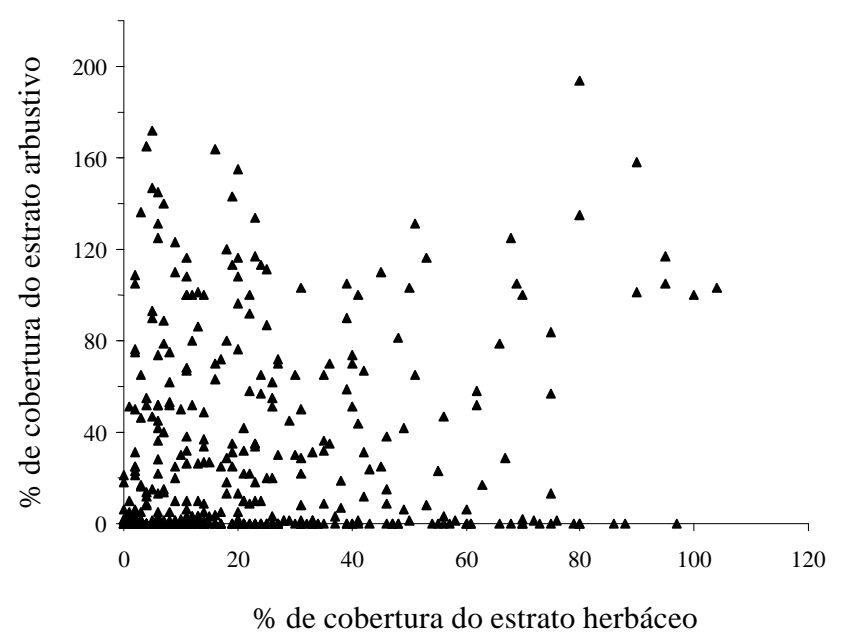

Figura 3. Correlação entre as percentagens de cobertura vegetal herbácea e arbustiva, estimadas em 600 parcelas de $1 \mathrm{~m}^{2}$ na formação aberta de Clusia do Parque Nacional da Restinga de Jurubatiba $\left(r_{s}=0,36, P<0,001\right)$. Em algumas parcelas o valor pode ser superior a $100 \%$ devido ao adensamento da vegetação e, conseqüente, sobreposição da copa das espécies amostradas.
Alguns estudos que comparam parâmetros estruturais de estratos herbáceo e arbustivo propõem que algumas espécies podem ser mais abundantes sob arbustos, enquanto outras teriam melhor desempenho em áreas sem cobertura vegetal arbustiva (Went 1942; Shmida \& Whittaker 1981; Silvertown \& Wilson1994; Tielbörger \& Kadmon 1997). Estas interações espécieespecíficas poderiam explicar a baixa correlação significativa observada para os dados de cobertura da Figura 3.

Considerando todos os resultados apresentados, pode-se concluir que, embora o estrato arbustivo seja fisionomicamente dominante na comunidade, este não parece determinar a maior ou menor ocorrência de espécies herbáceas. Por isso estudos sobre distribuição espacial e associação entre espécies, na formação aberta de Clusia, poderão esclarecer melhor a coexistência desses dois estratos.

\section{Agradecimentos}

Os autores agradecem à Leonora Cardin, aluna do Bacharelado em Botânica da UFRJ, e ao Sr. Joanito V. Azeredo, funcionário do Departamento de Botânica da UFRJ, pela inestimável colaboração na coleta dos dados; aos coordenadores e funcionários do Núcleo de Pesquisas Ecológicas de Macaé (NUPEM/UFRJ), pelo apoio e infraestrutura; aos demais membros do Laboratório de Ecologia Vegetal, Fabio Rubio Scarano e E.A.Mattos pelas sábias e constantes discussões que ajudaram a melhorar este manuscrito; ao CNPq, pelo apoio financeiro por meio do Programa de Pesquisas Ecológicas de Longa Duração (PELD/Site 5).

\section{Referências bibliográficas}

Almeida, A.L. \& Araujo, D.S.D. 1997. Comunidades vegetais do cordão arenoso externo da Reserva Ecológica Estadual de Jacarepiá, Saquarema, RJ. Oecologia Brasiliensis 3: 47-63.

Araujo, D.S.D. 1992. Vegetation types of sandy coastal plains of tropical Brazil: a first approximation. Pp. 337-347. In: U. Seeliger (ed.). Coastal plant communities of Latin America. Academic Press, New York.

Araujo, D.S.D. 2000. Análise florística e fitogeográfica das restingas do Estado do Rio de Janeiro. Tese de Doutorado. Universidade Federal do Rio de Janeiro, Rio de Janeiro.

Araujo, D.S.D. \& Maciel, N.C. 1998. Restingas fluminenses: biodiversidade e preservação. Boletim da Fundação Brasileira para Conservação da Natureza 25: 27-51. 
Araujo, D.S.D. \& Peixoto, A.L. 1977. Renovação da comunidade vegetal de restinga após queimada. Pp. 1-18. In: Anais do XXVII Congresso Nacional de Botânica. Academia Brasileira de Ciências. Rio de Janeiro.

Araujo, D.S.D; Pereira, M.C.A. \& Pimentel, M.C.P. 2004. Flora e estrutura de comunidades no Parque Nacional da Restinga de Jurubatiba - Síntese dos conhecimentos com enfoque especial para formação aberta de Clusia. Pp. 59-76. In: C.F.D. Rocha; F.A. Esteves \& F.R. Scarano (eds.). Pesquisas de longa duração na Restinga de Jurubatiba: ecologia, história e conservação. Rima Editora, São Carlos.

Araujo, D.S.D; Scarano, F.R.; SÁ, C.F.C.; Kurtz, B.C.; Zaluar, H.L.T.; Montezuma, R.C.M. \& Oliveira, R.C. 1998. As comunidades vegetais do Parque Nacional da Restinga de Jurubatiba, Macaé, RJ. Pp. 39-62. In: F.A. Esteves (ed.). Ecologia das lagoas costeiras do Parque Nacional de Jurubatiba e do município de Macaé. UFRJ, Rio de Janeiro.

Assumpção, J. \& Nascimento, M.T. 1998. Fitofisionomia de uma restinga no extremo norte do litoral fluminense: um novo elemento no mosaico? Pp. 158-164. In: S. Watanabe (coord.). Anais do IV Simpósio de Ecossistemas Brasileiros, v.3, ACIESP, São Paulo.

Assumpção, J. \& Nascimento, M.T. 2000. Estrutura e composição florística de quatro formações vegetais de restinga no complexo lagunar Grussaí/Iquipari, São João da Barra, RJ, Brasil. Acta Botanica Brasilica 14(3): 301-315.

Brower, J.E. \& Zar, J.H. 1984. Field \& laboratory methods for general ecology. $2^{\text {nd }}$ ed. W.C. Brown Company Publishers, Dubuque, Iowa.

Brummitt, R.K. \& Powell, C.E. 1992. Authors of plant names. Royal Botanical Gardens, Kew.

Cirne, P.; Zaluar, H.L.T. \& Scarano, F.R. Plant diversity, interspecific associations and postfire resprouting on a sandy spit in Brazilian coastal plain. Ecotropica 9: 33-38.

Citadini-Zanette, V. 1984. Composição florística e fitossociológica da vegetação herbácea terrícola de uma mata de Torres, Rio Grande do Sul, Brasil. Iheringia 32: 23-62.

Citadini-Zanette, V. \& Baptista, L.R.M. 1989. Vegetação herbácea terrícola de uma comunidade florestal em Limoeiro, município de Torres, Rio Grande do Sul, Brasil. Boletim do Instituto de Biociências da Universidade Federal do Rio Grande do Sul 45: 1-87.

Embrapa Monitoramento por Satelite, consultado em 11/02/2004 www.cdbrasil.cnpm.embrapa.br.

Gaston, K.J. \& Lawton, J. H. 1990. Effects of scale and habitat on the relationship between regional distribution and local abundance. Oikos 58: 329-335.

Gentry, A.H. \& Dodson, C.H. 1987. Diversity and biogeography of Neotropical vascular epiphytes. Annals of the Missouri Botanical Garden 75: 1-34.

Henriques, R.P.B.; Araujo, D.S.D. \& Hay, J.D. 1986. Descrição e classificação dos tipos de vegetação da restinga de Carapebus, Rio de Janeiro. Revista Brasileira de Botânica 9(2): 173-189.
Henriques, R.P.B.; Meireles, M.L. \& Hay, J.D. 1984. Ordenação e distribuição de espécies das comunidades vegetais na praia da restinga de Barra de Maricá, Rio de Janeiro Revista Brasileira de Botânica 7: 27-36.

Magurran, A.E. 1988. Ecological diversity and its measurement. Princeton University Press, New Jersey.

Martin, L.; Suguio, K. \& Flexor, J.M. 1993. As flutuações de nível do mar durante o quaternário superior e a evolução geológica de "deltas" brasileiros. Boletim do Instituto de Geociências-USP, Publicação Especial, 15: 1-186.

Mattos, E.A; Braz, M.I.G.; Cavalin, P.O.; Rosado, B.H.P.; Gomes, J.M.; Martins, L.S.T. \& Arruda, R.C.O. 2004. Variação Espacial e Temporal em Parâmetros Fisioecológicos de Plantas. Pp. 99-116. In: C.F.D. Rocha; F.A. Esteves \& F.R. Scarano (eds.). Pesquisas de longa duração na Restinga de Jurubatiba: ecologia, história e conservação. Rima Editora, São Carlos.

Menezes, L.F.T. \& Araujo, D.S.D. 1999. Estrutura de duas formações vegetais do cordão externo da restinga de Marambaia, RJ. Acta Botanica Brasilica 13(2): 223-235.

Müller, S.C. 1999. Estrutura sinusial e relações florísticas dos componentes herbáceo e arbustivo de uma floresta costeira subtropical. Dissertação de Mestrado. Universidade Federal do Rio Grande do Sul, Porto Alegre.

Müeller-Dombois, D. \& Ellenberg, H. 1974. Aims and methods of vegetation ecology. John Wiley \& Sons, New York.

Oliveira-Galvão, A.L.C.; Galvão, W.S. \& Carvalho, V.C. 1990. Monitoramento da cobertura vegetal da restinga de Carapebus-Macaé (RJ), a partir de imagens orbitais. Pp. 442-454. In: S. Watanabe (coord.). II Simpósio de Ecossistemas da Costa Sul e Sudeste Brasileira: Estrutura, função e manejo, v.3, ACIESP, São Paulo.

Ormond, W.T. 1960. Ecologia das restingas do Sudeste do Brasil. Comunidades vegetais das praias arenosas. Arquivos do Museu Nacional 50: 185-236.

Sá, C.F.C. 1996. Regeneração em área de floresta de restinga na Reserva Ecológica Estadual de Jacarepiá, Saquarema, RJ: I - estrato herbáceo. Arquivos do Jardim Botânico do Rio de Janeiro 34(1): 177-192.

Scarano, F.R. 2002. Structure, function and floristic relantioships of plants communities in stressful habitats marginal to Brazilian Atlantic Rainforest. Annals of Botany 90: 517-524.

Scarano, F.R.; Duarte, H.M.; Ribeiro, K.T.; Rodrigues, P.J.F.P. \& Barcellos, E.M.B. 2001. Four sites with contrasting environmental stress in southeastern Brazil: relations of species, life form diversity and geographic distribution to ecophysiological parameters. Botanical Journal of the Linnean Socieity 136: 345-364.

Silvertown, J. \& Wilson, J.B. 1994. Community structure in a desert perennial community. Ecology 75: 409-417.

Shmida, A. \& Whittaker, R.H. 1981. Pattern and biological microssite effects in two shrub communities, southern California. Ecology 62: 243-251

Tielbörger, K. \& Kadmon, R. 1997. Relationships between shrubs and annual communities in a sandy desert ecosystem: a three-year study. Plant Ecology 130: 191-201. 
Valentin, J.L. 2000. Ecologia Numérica: uma introdução à análise multivariada de dados ecológicos. Ed. Interciência Ltda, Rio de Janeiro.

Went, F.W. 1942. The dependence of certain annual plants on shrubs in southern California deserts. Bulletin of the Torrey Botanical Club 69(2): 100-114.
Zaluar, H.L.T. \& Scarano, F.R. 2000. Facilitação em restingas de moitas: Um século de buscas por espécies focais. Pp. 3-23. In: F.A. Esteves \& L.D. Lacerda (eds.). Ecologia de restingas e lagoas costeiras. NUPEM/UFRJ, Rio de Janeiro.

Zar, J.H. 1996. Biostatistical Analysis. 3 ed. Prentice-Hall Inc, New Jersey. 